\title{
When similarities matter more than differences: a reply to Wilson et al.
}

\author{
Benjamin D. Hoffmann', Franck Courchamp ${ }^{2}$ \\ I CSIRO, Tropical Ecosystems Research Centre, PMB 44, Winnellie, Northern Territory, Australia, 0822 \\ 2 Ecologie, Systématique \& Evolution, University of Paris Sud, CNRS, AgroParisTech, Université Paris-Saclay, \\ Orsay Cedex 91405, France
}

Corresponding author: Benjamin D. Hoffmann (Ben.Hoffmann@csiro.au)

Academic editor: IngolfKühn | Received 25 August 2016 | Accepted 31 August 2016 | Published 14 September 2016

Citation: Hoffmann BD, Courchamp F (2016) When similarities matter more than differences: a reply to Wilson et al. NeoBiota 31: 99-104. doi: 10.3897/neobiota.31.10290

\begin{abstract}
In our recent Discussion paper, we presented our view that the only real distinction between biological invasions and natural colonisations is the human element. We agree that invasion science is a very important science, not only to better understand the role that human mediation plays for colonisation, but also for many other science fields. We agree with all invasion researchers that the human influence can result in spectacular differences, including in rates of species movement, rates of successful colonisation, the particular species being moved, the biogeography of dispersal pathways and rates of any resulting ecological disturbance and biodiversity loss. Our deep point is that that species dispersed by human-mediation or natural colonisation are all subject to the same basic laws and rules of ecology, identical to many other phenomenon that occur naturally and can be greatly influenced by people. The human dimension is merely a mechanistic distinction, albeit important because it exposes insights about the colonisation process that cannot be seen by the study of natural colonisations alone. We provide 10 hypotheses that can be scientifically tested to determine whether biological invasions and natural colonisations are two separate processes or the same process being influenced by different mechanisms.
\end{abstract}

\section{Keywords}

Alien, biological invasion, colonisation, dispersal, exotic, invasion, introduction 


\section{Humans have a huge influence on many phenomena}

In our recent paper (Hoffmann and Courchamp 2016) we point out at that despite many obvious differences between human-mediated biological invasions and natural colonisation, there are also many similarities. Ultimately, yes, they have differences, no one denies that, but are they THAT different? In a reply, Wilson et al (2016) have focused on the differences to argue the point that they are distinct, concluding biological invasions and natural colonisations are very often different; sometimes this matters, sometimes it does not. Here we respond to their arguments, ultimately concluding that biological invasions and natural colonisations are very often similar; sometimes this matters, sometimes it does not. In doing so, we further reinforce that the concepts in invasion ecology should not be artificially dissociated from general ecology.

The deep intent of our original paper was to argue that species dispersed by humanmediation or by natural colonisation are all subject to the same laws of ecology and that overlooking these similarities could slow down scientific progresses in invasion biology. As succinctly put by Lawton (1999) "The most useful scientific laws yield deep insights into the workings of nature: rules are less grand. Mechanisms are weaker still: a mechanism or mechanisms can generate unique phenomena: but general rules require common mechanisms". Undoubtedly the mechanism of human-mediated dispersal generates the unique phenomenon of modern biological invasions, but species dispersed by the two transport modes are subject to the same ecological laws and rules. To cite Jeschke (2014) "a look at the concepts and hypotheses in invasion ecology reveals that these are deeply connected with those in general ecology." The two fields have essentially the same hypotheses but with nuanced terminology, (eg invader vs coloniser). Their respective hypotheses, when boiled down to basics, are identical. Would anybody dare argue that hypotheses of propagule pressure, biotic resistance and enemy release only apply to species dispersed by human mediation but not natural colonisation? The fields and their hypotheses are not THAT different, because they focus on the same processes subject to the same biological laws and rules within a general theory of ecology (Scheiner and Willig 2008).

We cannot understate how important research about biological invasions have been to understanding colonisation, because it adds the other side of the continuum that has been studied by island biogeography (MacArthur and Wilson 1967). The commonalities between invasion science and general ecology have long been noted by many (Thompson et al.1995; Davis et al. 2001; Economo and Sarnat 2012). We agree that the human dimensions of biological invasions are pivotal to invasion biology, with clear implications for management and policy. But we are arguing that humanmediation is a mechanism, and thus essentially a biological invasion is a sub-class of the process of colonisation. Equally, natural colonisation is also sub-class of colonisation, and both are subject to the same natural laws.

We agree with Wilson et al. (2016) that scientific testing of hypotheses should determine whether colonisation and biological invasions are truly unique, or just the same overall process initiated by different mechanisms. Naturally there would be huge differences if the focus was at the level of mechanisms such as pathways (i.e. propagule 
pressure for most species is undoubtedly statistically significantly different between human-mediated dispersal and self-dispersal). We argue that this is not the correct level for a fundamental scientific distinction, but instead the difference should lie much deeper at the level of laws and rules. As a suggestion of how to test this, we refer to the first 10 hypotheses of general ecology explored in Prins and Gordon (2014). Although specifically written for biological invasions, if the terms for biological invasions and natural colonisations were interchanged in each hypothesis, the hypotheses would be identical for both invasion biology and general ecology.

Hypothesis 1: A species will not be able to invade/colonise an area that has abiotic conditions that are outside its physiological tolerance levels.

Hypothesis 2: The extent of an invasion/colonisation is negatively correlated to species diversity of functional guild competitors in the invaded environment.

Hypothesis 3: An invasive/colonising species will not be able to replace a native species if they occupy the same niche and are in all other ways equal.

Hypothesis 4: A species will not be able to invade/colonise an area that harbours pathogens (that cause disease) or predators (that prey on the invading species) that it has not encountered before.

Hypothesis 5: A species will not be able to invade/colonise an area if its co-evolutionary species (necessary for parts of the invader's life cycle) is/are not present in the area. Hypothesis 6: Species that occur at low population densities in their native range will not be invasive/prone to colonise.

Hypothesis 7: A species will not be able to invade/colonise an area if it has a lower use efficiency of its limiting resource than a native species that occupies the same location. Hypothesis 8: Species can more easily invade/colonise highly disturbed areas; this disturbance can be either man-made or natural.

Hypothesis 9: Species from older lineages are more vulnerable to being replaced by invasive/colonising species that occupy a similar niche.

Hypothesis 10: A species will only be able to invade/colonise an area if it has a lifehistory strategy which is more r-selected (or 'weedy') that that of the species which already is occupying the niche.

The testing of the hypotheses for this purpose is not to determine if individual hypotheses should be rejected or not (for an extremely interesting insight into this refer to Prins and Gordon 2014), but instead to determine if there is a difference in the rejection of the null hypothesis for individual hypotheses between the two species movements. If the pattern of acceptance/rejection of these hypotheses is the same between species dispersed by human-mediation vs self-dispersal, then we would have to conclude that species dispersing by the two transport modes are subject to the same process with the same underlying ecological laws.

We agree with Wilson et al. (2016) on the differences of mechanisms (including the degree [rate] at which they occur), as well as their implications, especially for management and policy. But absolutely nowhere have Wilson et al. (2016) demonstrated that the two are subject to a different process. Specifically they give an example 
about an apparent difference between the creation of the stones at Stonehenge and the smooth pebbles on a rocky shore. One is obviously human-mediated, the other natural. We agree that the mechanism(s) that gave rise to the rocks being modified into other shapes and configurations is different, but we argue that the underlying laws dictating the erosion process (e.g. laws of thermodynamics and the physics of friction) are identical for both. Indeed humans could create a beach of round pebbles, and there is absolutely nothing in the Laws of Thermodynamics preventing Stonehenge from naturally forming, but the probability (and hence rate) of it occurring is extremely low.

An important point that we want to make clear again is that we by no means are saying that invasion science is redundant. It is a vital science focused on species that are dispersed by people, and forms part of a family of sciences around biology and ecology with wideranging linkages with many other sciences including epidemiology and conservation sciences. This seems to be repeatedly lost in arguments by Wilson et al. (2016). For example we fully recognise the fields of conservation biology and population biology. But rather than seeing a black-and-white distinction based on differences of susceptibility of populations to stochastic events relative to population sizes, we instead see that this demonstrates that population sizes follow a continuum, which partly determines population's relative susceptibility to stochastic events. Our argument is one of a holistic view that whether a population is studied by either science, the process(es) underlying susceptibility to a stochastic event are the same, all that differs is the degree to which a population is susceptible.

Wilson et al. (2016) presented numerous arguments to demonstrate that the magnitude of differences of the two transport modes is an important science distinction, particularly noting that differences are often not just a linear change in rate, but a "step change". The influence of people on climate change was also provided as an example of such a "step change", implying some important science distinction. We find the climate change argument presented by Wilson et al. (2016) is instead very pertinent to our argument because of the identical nature of it with the holistic view of colonisation (Figure 1). Are the authors suggesting that the processes that influence global climates will change differently if the $\mathrm{CO}_{2}$ is naturally released or human induced? Climate scientists agree that increasing $\mathrm{CO}_{2}$ levels in the atmosphere is resulting in climate change. Climate scientists agree that there are human-mediated and natural contributions to global atmospheric $\mathrm{CO}_{2}$. Much climate science also focuses solely on the human-mediated component. Despite there being big differences between the rate of $\mathrm{CO}_{2}$ release, the mechanisms by which $\mathrm{CO}_{2}$ is released, and the geography of $\mathrm{CO}_{2}$ release, for the two modes of atmospheric $\mathrm{CO}_{2}$ accumulation no climate scientist would argue that there is some fundamental difference in implications for projected climate changes due to the human-mediated or natural source of $\mathrm{CO}_{2}$. Certainly, whilst there are marked implications for management of the different sources of emissions, none are advocating for a separate science or that there are different processes at play. Similarly, if a significant volcanic eruption somehow induced an atmospheric $\mathrm{CO}_{2}$ stepchange, are Wilson et al. (2016) arguing that the laws of chemistry and thermodynamics would influence the climate differently to an identical human-induced step-change? Ultimately, for all of their examples claiming that rates and degree differ, not a single difference in underlying process was given. 

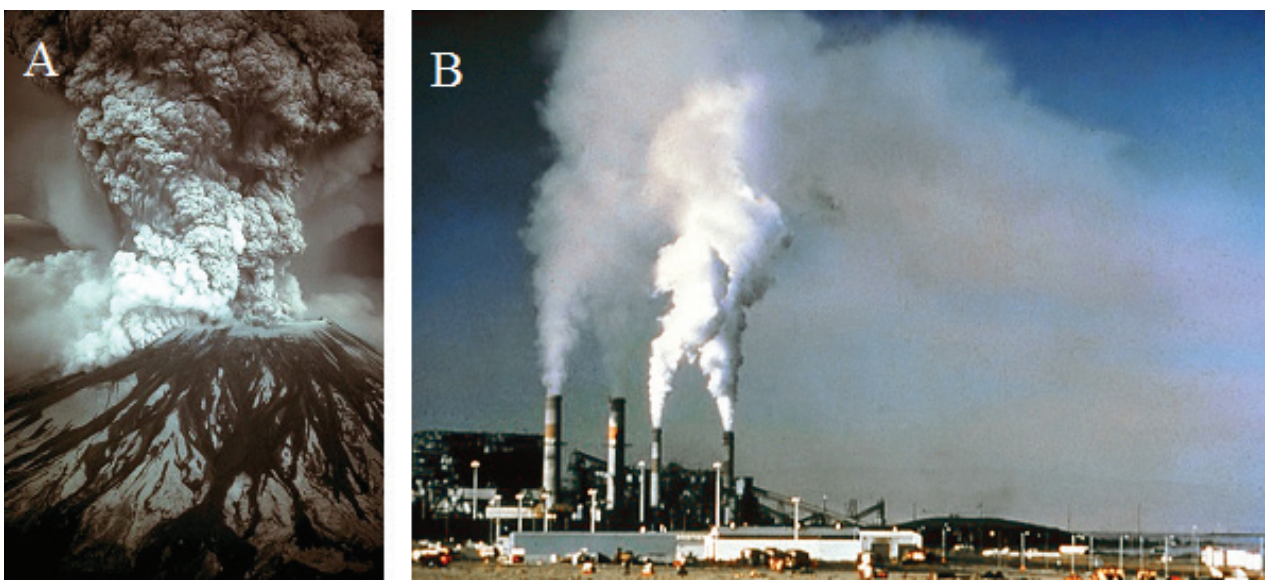

Figure I. The process of climate change can be influenced by atmospheric composition change from natural sources such as volcanoes $\mathbf{A}$ or human-mediated sources such as electricity power stations $\mathbf{B}$ Clearly the two mechanisms of gas release have many significant differences, including the origins of the gases, nuanced sciences, rates, and policy implications, but their influence on the process of climate change is governed by identical laws of chemistry and thermodynamics. When considered holistically in the context of climate change, the two mechanisms are not THAT different, and are not two separate processes. $\mathbf{A}$ is courtesy of the U.S. Geological Survey, and B is courtesy of the U.S. National Parks Service.

We restrict comment about impacts here, just as we did previously, because impacts are not part of the colonisation process, but can be a consequence of it. For this reason, impacts were also not included in Blackburn's framework (Blackburn et al. 2011). We agree that some invasive species have created much extinction, and have done so spectacularly. The intent of our text was twofold: first to point out that most species moved by people have not been unambiguously shown by science to induce extinction. Indeed, only about 30 taxa are implicated with extinctions (Bellard et al. 2016), and therefore extinction is an exception, not a rule. Second, extinction as a result of colonisation is historically not confined just to species dispersed by people (Bellard et al. 2016), and as also stated by Wilson et al. (2016) nor is it likely "to be just a modern phenomenon".

We agree with Wilson et al. (2016) that, "at the heart of invasion science is the realisation that ... the human dimension of invasions is a fundamental component in the social-ecological systems in which invasions need to be understood and managed." Naturally, without the human component there could be no human-mediated dispersal. But that is as deep as it gets. Without denying the pivotal role of humans in invasion biology, one must keep in mind that the biological, or ecological, component of it is at least, if not more, important. Even Wilson et al. (2016) wrote "While conceptually the same barriers are present, the resources provided for establishment mean that some barriers are rendered inconsequential." We couldn't agree more. Humans override some barriers by overcoming the limitation of the process that affect colonisation - species self-dispersing have to overcome the same barriers by themselves. Invasion science asks the focused question, how do people influence colonisation, and how do those influences change colonisation outcomes? 
Ultimately, despite the statement by Wilson et al. (2016) "we strongly believe that the answer is yes: biological invasions and natural colonisations differ in processes and mechanisms" not a single process was presented, there or elsewhere. Let science be the judge. If there is indeed some deep and fundamental science difference between species undergoing range expansion by the two transport modes, then an underlying biological law needs to be found that applies to only one. To conclude along the same lines as Wilson et al. (2016), we believe that future civilisations will recognise from the geological record that at the turn of the Anthropocene, the major mechanisms driving the colonisation process for species globally changed from those that are "natural" and largely random and slow, to those that are driven by a dominant species (humans) and are largely non-random and fast.

\section{References}

Bellard C, Cassey P, Blackburn TM (2016) Alien species as a driver of recent extinctions. Biology Letters 12: 4. doi: 10.1098/rsbl.2015.0623

Blackburn TM, Pyšek P, Bacher S, Carlton JT, Duncan RP, Jarošík V, Wilson JRU, Richardson DM (2011) A proposed unified framework for biological invasions. Trends in Ecology and Evolution 26: 333-339. doi: 10.1016/j.tree.2011.03.023

Davis MA, Thompson K, Grime JP (2001) Charles S. Elton and the dissociation of invasion ecology from the rest of ecology. Diversity and Distributions 7: 22-40. doi: 10.1046/j.14724642.2001.00099.x

Economo EP, Sarnat EM (2012) Revisiting the ants of Melanesia and the taxon cycle: historical and human-mediated invasions of a tropical archipelago. The American Naturalist 180. doi: $10.1086 / 66596$

Hoffmann BD, Courchamp F (2016) Biological invasions and natural colonisations: are they that different? NeoBiota 29: 1-14. doi: 10.3897/neobiota.29.6959

Jeschke JM (2014) General hypotheses in invasion ecology. Diversity and Distributions 20: 1229-1234. doi: 10.1111/ddi.12258

Lawton JH (1999) Are there general laws in ecology? Oikos 84: 177-192. doi: 10.2307/3546712

MacArthur TH, Wilson EO (1967) The theory of Island biogeography. Princeton University Press, Princeton, NJ.

Prins HHT, Gordon IJ (2014) Invasion biology and ecological theory: Insights from a continent in transformation. Cambridge University Press, 528 pp. doi: 10.1017/CBO9781139565424

Scheiner SM, Willig MR (2008) A general theory of ecology. Theoretical Ecology 1: 21-28. doi: 10.1007/s12080-007-0002-0

Thompson K, Hodgson JG, Rich TCG (1995) Native and alien invasive plants: more of the same? Ecography 18: 390-402. doi: 10.1111/j.1600-0587.1995.tb00142.x

Wilson JRU, García-Díaz P, Cassey P, Richardson DM, Pyšek P, Blackburn TM (2016) Biological invasions and natural colonisations are different - the need for invasion science. NeoBiota 31: 87-98. doi: 10.3897/neobiota.31.9185 